\title{
Qué decir cuando te preguntan ¿qué tal? Saludos en clase de ELE para hablantes de polaco ${ }^{1}$
}

\author{
DOROTA KOTWICA
}

Universidad de Alcalá de Henares; Universitat de València

dorota.kotwica@uv.es

\begin{abstract}
Resumen: Este artículo trata de las cuestiones socioculturales relacionadas con los saludos coloquiales con pregunta por la salud (¿qué tal?, ¿cómo estás?). Se pretende cumplir un doble objetivo: en primer lugar, desde el punto de vista teórico, se ofrece una reseña de las diferencias básicas de este tipo de saludos en español y en polaco. En segundo lugar, desde el punto de vista aplicado, se ofrece una secuencia de actividades prácticas orientadas a mejorar la competencia comunicativa de los alumnos con un nivel B1. La propuesta promueve la observación y la reflexión acerca de las reglas que rigen el empleo de las preguntas por la salud en español. Para ello, se utilizan muestras de textos (orales y escritos) producidos por los hablantes nativos.
\end{abstract}

Palabras clave: competencia comunicativa, competencia sociocultural, saludos, polaco.

\section{What should you say if they ask ¿qué tal? Greetings in SLE class for Polish speakers}

Abstract: This paper focuses on sociocultural aspects of colloquial greetings in Spanish accompanied by a question about health (¿qué tal?, ¿cómo estás? 'how are you?'). The aim of this paper is twofold: first, a theoretical overview is offered of the basic differences between greetings in Spanish and in Polish. Second, a sequence of practical activities is described. They are designed to improve the communicative competence of students at B1 level. The proposal encourages observation and reflection on the rules that govern the use of questions in Spanish greetings. For this, samples of texts (oral and written) produced by native speakers are used.

Key words: communicative competence, sociopragmatic competence, interculturality, greetings, Polish.

\section{Introducción}

Los alumnos de ELE aprenden las fórmulas básicas de saludo y despedida en las primeras clases y es difícil imaginar un manual de lengua de nivel básico en el que no se trate este tema. Sin embargo, como señala Zieliński (2018), en la enseñanza de español a los polacohablantes y en la enseñanza de polaco a los hispanohablantes no se presta suficiente atención a los diferentes factores socioculturales relacionados con los saludos, «quizá porque se piense que esas primeras expresiones que adquieren parten del mismo

\footnotetext{
${ }^{1}$ Este trabajo se ha realizado en el marco del proyecto postdoctoral APOSTD/2019/161 financiado por la Generalitat Valenciana y el Fondo Social Europeo. También ha contado con el apoyo del proyecto Es.Vag.Atenuación: La atenuación pragmática en su variación genérica: géneros discursivos escritos y orales en el español de España y América (referencia FFI2016-75249-P).
} 
componente pragmático y formal e implican el mismo funcionamiento en los códigos de ambas lenguas» (Zieliński, 2018: 105). Sin embargo, está comprobado que existen varias diferencias entre cómo se saluda en español y en polaco, y estas no siempre se atienden de manera adecuada en clase. En consecuencia, a menudo surgen choques o malentendidos culturales y lingüísticos que reportan los polacos que empiezan a relacionarse con los españoles después de haber aprendido español fuera de España. Para resolver este problema es necesario, en primer lugar, explicar de qué clase de diferencias se trata $\mathrm{y}$, en segundo lugar, tratar de darle alguna solución desde la didáctica del ELE. Nos ocuparemos de ello en este trabajo.

\section{Las fórmulas de saludo en español y en polaco: ¿dónde están las diferencias?}

Las diferencias entre cómo se saluda en polaco y en español son múltiples y se relacionan, por ejemplo, con las situaciones y los espacios en los que se saluda o no, la formalidad, etc. Sin embargo, en esta ocasión nos ocuparemos únicamente de una cuestión muy concreta: el funcionamiento de los saludos con preguntas por la salud, en un contexto de comunicación informal.

En español, algunas preguntas por la salud suelen ser rutinarias y no requieren una respuesta concreta, ya que, al igual que los enunciados de saludo y despedida, «pertenecen al discurso repetido: son modismos descargados de su significación primitiva para cumplir una función puramente pragmática» (Moreno Fernández, 1986: 267). Lo que ocurre es que los polacos interpretan estas fórmulas españolas de manera literal y esto es fruto de una «la clara transferencia sociopragmática del polaco» (Zieliński, 2018: 106; véase también Jaworski (1994) sobre las diferencias entre el polaco y el inglés):

Esto se debe a que las expresiones que giran en torno al bienestar o a la salud del destinatario carecen de carácter ritual o formulario en polaco. Es decir, al no poseer carácter convencional, el destinatario polaco tiende a descodificar las respectivas expresiones polacas (jak się masz? ‘¿cómo estás?' Jak leci? ‘ccómo te va?’ Jak tam? ‘¿qué tal?’, etc.) como actos de habla a través de los cuales el emisor se interesa realmente por el bienestar de un destinatario que ya conoce. (Zieliński, 2018: 106-107)

Además, las preguntas, ¿qué tal? y ¿cómo estás? normalmente exigen, por defecto, una respuesta positiva, aunque no se corresponda con el estado real de la persona:

El hablante quiere a toda costa obtener del interlocutor una contestación favorable. Todos estos giros y aún más el descolorido «¿qué tal?», con la correspondiente contestación pura «bien, gracias, ¿y usted?», están gastados hasta tal punto que no se sienten como pleonasmos o contradicciones, pues es frecuente el caso en que el interlocutor, requerido con más insistencia por su salud, a pesar del «bien, gracias» precedente, manifiesta un sentido y larga retahíla de quejas y lamentaciones. (Moya Corral y García Wiedemann, 1994: 400)

Por el contrario, la respuesta típica a las preguntas por la salud en la cultura polaca es «poco optimista», como señala Marcjanik (2013: 26), por ejemplo: jakoś leci 'así vamos', nic nowego 'nada nuevo', stara bieda 'lo de siempre' (bieda lit. 'miseria, pobreza'), lepiej nie mówić 'mejor ni te cuento'. A partir de la observación de las tendencias entre la generación más joven, Marcjanik (2013: 26) constata que cuando los polacos jóvenes emplean respuestas más positivas y optimistas (como wszystko $w$ porzadku 'todo bien', świetnie 'genial') es por una especie de estrategia o juego comunicativo consciente. 
Las diferencias en los supuestos socioculturales y pragmáticos relacionados con los saludos ocasionan malentendidos y choques culturales. Hemos confirmado en numerosas ocasiones el desconcierto de los alumnos polacos que contaban con asombro como, durante las primeras semanas de su estancia en España, los conocidos españoles les saludaban con un hola, ¿qué tal? y seguían su camino, sin esperar la respuesta o pararse a conversar. Y es que, en la cultura polaca, las preguntas por la salud suelen aparecer en las fórmulas de «saludos de apertura» que inician una interacción conversacional (Goffman, 1979) y no tanto en los «saludos de paso» que surgen cuando dos hablantes se cruzan en la calle o en un pasillo. Si dos polacos (conocidos, vecinos, compañeros de trabajo) se cruzan en algún lugar público, intercambian un saludo cortés, pero no preguntan por la salud del otro. La aparición de esta pregunta entre las personas que no se conocen mucho resultaría extraña y, entre las personas con una relación más cercana, iniciaría seguramente un intercambio más largo para no dejar la pregunta sin contestar.

Otra situación de sorpresa suele producirse cuando los polacos presencian intercambios breves entre los nativos en los que la pregunta por la salud se responde con otra pregunta similar, sin realmente dar ninguna información sobre cómo se encuentra uno, como en el ejemplo (1):

(1) A: Hola ¿qué tal?

B: ¿Qué hay?

De nuevo, lo que causa el choque es que no se responde a la pregunta. De hecho, en la lengua polaca existe el siguiente dicho popular: nie odpowiada sie pytaniem na pytanie ('no se responde a una pregunta con otra pregunta'). Como explica la especialista en la cortesía polaca, la profesora Marcjanik en el consultorio de la lengua (Poradnia Językowa $\mathrm{PWN}^{2}$ ) es inaceptable, desde el punto de vista de la cortesía polaca, contestar con una pregunta a otra pregunta de carácter cortés. El ejemplo que cita Marcjanik es precisamente el del saludo: puesto que la cortesía polaca está basada en la simetría, requiere que la muestra de interés sea correspondida (es decir, hay que contestar a la pregunta y después hacer la pregunta análoga sobre la salud del otro). Como apunta Marcjanik en la consulta citada, «La pregunta en vez de la respuesta muestra un desprecio hacia la postura empática del emisor de la primera pregunta» ${ }^{3}$.

Sin duda, la experiencia de convivencia con los nativos hispanohablantes ayuda a comprender las diferencias socioculturales y a mitigar la transferencia del polaco. Pero, obviamente, no todos los aprendices van a tener este tipo de experiencias y, además, «[c]omo profesores de lengua, no podemos conformarnos con que nuestros estudiantes se encuentren con los problemas cuando se desplacen al país de la lengua meta» (Miquel, 2004 s.p.). Asimismo, ofrecemos, a continuación, una propuesta práctica de cómo podría profundizarse en la explicación del saludo en español en una clase de ELE en un nivel B1.

\section{El planteamiento de las actividades}

\footnotetext{
${ }^{2}$ La consulta se encuentra disponible en línea en la dirección: https://sjp.pwn.pl/poradnia/haslo/pytaniem-napytanie;9970.html (consultado el 15 de abril de 2020).

${ }^{3}$ Nuestra traducción del original: «Pytanie zamiast odpowiedzi świadczy o lekceważeniu świadczącej o empatii postawy nadawcy pierwszego pytania».
} 
Las actividades que se han diseñado están relacionadas con la competencia comunicativa y dentro de ella, la sociolingüística que, según Miquel (2004), «ha venido siendo definida como el conocimiento de las reglas sociolingüísticas y culturales que hacen que las contribuciones sean apropiadas a los contextos». En concreto, se centran en un aspecto del acto «saludo informal» que, si bien existe en ambas culturas (polaca y española), no se relaciona con los mismas «presuposiciones culturales» (Miquel, 2004, s.p.). En algunas de las actividades propuestas no huimos de hacer la comparación con la lengua materna de los alumnos, puesto que ayuda a reforzar la autorreflexión y la adquisición de la competencia intercultural para que se conviertan en «usuarios conscientes de su propia y de la segunda lengua» (Palion-Musioł y Tatoj, 2016: 276).

Para el desarrollo de las actividades, hemos seleccionado varias muestras de materiales reales procedentes de la comunicación oral y escrita entre los hablantes nativos de español. Los materiales se han obtenido de Internet (un vídeo y publicaciones en los foros) y permiten observar diversos fenómenos de la pragmática y del español coloquial. Las actividades conforman una secuencia, por lo que la adquisición y ampliación de conocimientos se realiza de manera gradual y coherente.

El cronograma orientativo para la realización de las cinco actividades propuestas se muestra en la Tabla 1.

\begin{tabular}{|l|c|}
\hline \multicolumn{1}{|l|}{ Actividad } & Duración (en minutos) \\
\hline Actividad 0. ¿Cómo saludas? & 5 \\
\hline Actividad 1. ¿Cómo está Berto? & 5 \\
\hline $\begin{array}{l}\text { Actividad 2. En el consultorio } \\
\text { de Berto }\end{array}$ & 35 \\
\hline $\begin{array}{l}\text { Actividad 3. ¿Qué tal? en los } \\
\text { foros }\end{array}$ & 20 \\
\hline $\begin{array}{l}\text { Actividad 4. ¿Te ha pasado } \\
\text { esto? }\end{array}$ & 25 \\
\hline & Total \\
\hline
\end{tabular}

Tabla 1. Cronograma de las actividades

La propuesta va dirigida a los alumnos de nacionalidad polaca con un nivel B1 de español, pero puede ser adaptada también a los alumnos de otras nacionalidades o grupos mixtos.

\section{Las actividades prácticas}

Actividad 0. ¿Cómo saludas? La primera actividad tiene como objetivo la autorreflexión y la introducción al tema que se va a tratar. Se realiza en formato de cuestionario individual en el que aparecen las siguientes preguntas:

1. Cuando saludas a un amigo o a un conocido polaco ¿le preguntas cómo está?

2. ¿Harías esta pregunta a un taxista, a un dependiente en la tienda o a un vecino 
que encuentras en el ascensor?

3. ¿Cómo se responde normalmente a esta pregunta en polaco?

4. ¿Te resultaría extraño que alguien te contestara que se encuentra fatal o que ha tenido un mal día?

5. ¿Crees que un español contestaría igual que tú a las preguntas 1-4? ¿Qué diferencias habría?

Los alumnos comparan sus respuestas y observan si han contestado de manera similar (si vienen del mismo país, debería haber bastante consenso). El profesor avisa que el resto de las actividades se relaciona con estas mismas preguntas, pero desde el punto de vista de la cultura española.

Actividad 1. ¿Cómo está Berto? Se trata de una actividad de calentamiento muy breve. Los alumnos deben completar una sencilla conversación entre Ana y Berto, de acuerdo con lo que les sugieren los emoticonos en la segunda fila de la tabla (unos indican un estado de salud o ánimo positivo, otros, negativo). Después, por parejas, los alumnos comparan sus respuestas, pero de momento no se hace una puesta en común. Necesitaremos consultar sus respuestas más adelante, así que las guardan hasta entonces.

\begin{tabular}{|c|c|c|}
\hline Inicio de la conversación & \multicolumn{2}{|c|}{ Ana: Hola, Berto, ¿qué tal?, ¿cómo estás? } \\
\hline Cómo se siente Berto & 级 & $\sqrt{3} \cdot 0$ \\
\hline Completa la conversación & $\begin{array}{l}\text { Berto: } \ldots \ldots \ldots \ldots \ldots \ldots \ldots \ldots \ldots \\
\text { Ana: } \ldots \ldots \ldots \ldots \ldots \ldots \ldots \ldots \ldots \ldots \ldots\end{array}$ & 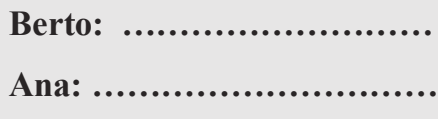 \\
\hline
\end{tabular}

Tabla 2. Ejercicio de calentamiento

Actividad 2. En el consultorio de Berto. En esta actividad se trabajará con un fragmento de vídeo del programa Late Motiv ${ }^{4}$. En concreto, se trata de un consultorio humorístico en el que Berto Romero contesta a las preguntas que la gente le hace llegar por las redes sociales. Sugerimos que se realicen por lo menos dos visionados, el primero sin la transcripción.

Como actividad de pre-visionado, para contextualizar el vídeo, el profesor enseña la captura de pantalla en la que aparecen Andreu Buenafuente y Berto Romero y una versión acortada de la pregunta a la que va a contestar Berto. Se explica la idea del consultorio y se presentan los dos actores, si los alumnos no los conocen. Los alumnos comentan entre ellos cómo creen que va a ser la respuesta de Berto. Pueden hacerlo en forma de una apuesta.

\footnotetext{
${ }^{4}$ Es un programa de televisión español presentado por Andreu Buenafuente. Se emite en \#0 de la plataforma Movistar+ (más información está disponible en línea, en http://latemotiv.com/late-motiv/).
} 


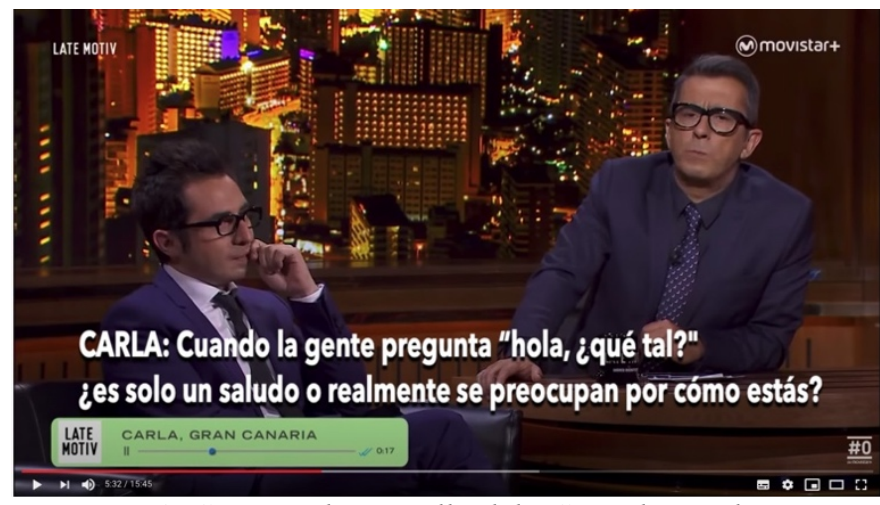

Imagen 1. Captura de pantalla del «Consultorio de Berto»

A continuación, se realizará el primer visionado del vídeo ${ }^{5}$ del que los alumnos tendrán que extraer únicamente información general. Después de ver el vídeo, se comentan las primeras reacciones y sensaciones (¿se han reído?, ¿se han sorprendido?). El profesor pide que levanten la mano los que antes de ver el vídeo han contestado bien a la pregunta (los que «han ganado la apuesta»).

Posteriormente, se realiza otro visionado del vídeo (o dos, si se considerará necesario). Esta vez, los alumnos reciben una transcripción. Con la transcripción se trabajará ya no solo la cuestión de los saludos, sino que también se hablará de algunos fenómenos del registro coloquial presentes en el fragmento. Puesto que los signos de transcripción utilizados son muy sencillos, proponemos que solo se avise de que no se han reproducido comas ni puntos y que la barra indica una pausa.

Aportamos la transcripción y las propuestas de las actividades a continuación:

LA CONSUlTA: hola Berto / hola Andreu / soy Carla de Gran Canaria // queríaa ver si me podían resolver una duda / cuando la gente pregunta hola ¿qué tal? / ¿es solo un saludo o realmente se preocupan por cómo estás? / es una duda que me llevaa atormentando durante años / muchas gracias / un saludo

BERTO: de nada de nada / mira // que- querida amiga NADIE quiere saber cómo estás / cuando / cuando dice hola ¿qué tal? nadie quiere saber cómo estás / es más / ni siquiera cuando preguntan ¿cómo estás? quieren saber cómo estás // eso se dice para que tú creas que al otro le importas / pero es n- no importas nada / es una pregunta que no espera respuesta y sobre todo NUNCA RESPUESTA NEGATIVA ¿vale? / nunca // mira / una vez me dio por responder con sinceridad / esto es cierto ¿vale? / esto ocurrió durante la peor época de no dormir cuando tuvimos a los gemelos ¿vale?

ANDREU: sí

BERTO: teníamos tres niños pequeños

ANDREU: tuvimos con tu mujer

BERTO: sí sí claro claro

ANDREU: es que me has miradoo

[RISAS DEL PÚBLICO]

BERTO: NO no no no

5 La grabación del capítulo de Late Motiv al que nos referimos se encuentra disponible en la plataforma YouTube, en la dirección: https://youtu.be/ormhwh1SaU0 . Se trata del capítulo 83 del programa, publicado en YouTube el 9 de junio de 2016. El fragmento concreto que nos interesa comienza a partir del minuto 5:27 y termina en el minuto 7:30. 
ANDREU: es que me has mirao

BERTO: NO NO NO no no

ANDREU: es que me has mirado al decir tuvimos a los gemelos / joer / me ha dado un escalofrío ¿sabes?

[RISAS DE BERTO]

ANDREU: perdón perdón perdón

BERTO: molaría que hubiéramos adoptado unos / pero para quedarnos uno cada uno

ANDREU: ss- sí sí

[RISAS DEL PÚBLICO]

BERTO: no / teníamos / el caso es que yo tenía tres niños pequeños en casa y NO dormíamos nada y me llegué a apuntar la conversación // es cierto ¿eh? / me lo apunté / fue tal y como la voy a leer

ANDREU: sí

BERTO: me viene una persona y me dice / había pasado un puente y me dice / ¿qué tal han ido las fiestas? / ¿bien? / y yo digo bueno imagínate con los tres niños / sin dormir / el follón / han estado enfermos // sin salir de casa en to'l puente / y me responde // bueno / pero en familia ¿no? / bien

[RISAS Y APLAUSOS DEL PÚBLICO]

BERTO: ¿vale? o sea

ANDREU: ya ya ya

BERTO: fíjate Carla que ni siquiera había preguntado ¿qué tal? sino ¿qué tal habían ido las fiestas? / era una pregunta concreta / y ni con esas le importaba / no le interesaba conocer la respuesta / ¿por qué? / porque la respuesta era mala / a nadie le interesa saber que estás mal / ¿cómo te atreves a compartir conmigo tu mierda? / así que Carla / aunque lleves el brazo colgando o te salgan las tripas por el culo / ¿cómo estás? / bien ¿y tú? / también / y a la calle

[RISAS DEL PÚBLICO]

En primer lugar, se trabaja sobre la comprensión del vídeo. Para ello, se utilizan las siguientes preguntas:

1. Según Berto, ¿por qué la gente hace la pregunta “¿qué tal?”?

2. Cuando Berto y su mujer tuvieron a los gemelos, ¿cómo se encontraban? ¿Qué problema tenían?

3. ¿Qué le pasó a Berto y a su familia durante los días anteriores a la conversación de la que habla? ¿Fueron días laborables?

4. ¿Respondió Berto sinceramente a la pregunta ¿qué tal han ido las fiestas?

5. ¿Aceptó la persona con la que habló Berto su respuesta?

6. ¿Por qué, según Berto, a esa persona no le importaba su respuesta? ¿Era un caso especial o es algo común?

7. ¿Por qué la gente se ríe de la historia de Berto?

8. ¿Qué recomienda responder Berto a la pregunta ¿cómo estás? en cualquier situación?

9. ¿Crees que nunca es posible contestar de otra manera? ¿De qué depende?

En segundo lugar, se ofrece un ejercicio relacionado con el vocabulario que potencialmente podría causarles problemas a los alumnos. Deben relacionar las palabras con sus significados en español. 


\begin{tabular}{|l|l|}
\hline PALABRA & DEFINICIÓN \\
\hline Tripas & Prestar la atención a algo. \\
\hline Importar & $\begin{array}{l}\text { Sensación producida por una emoción in } \\
\text { especialmente de terror. }\end{array}$ \\
\hline Escalofrío & Tener una cosa o persona interés para otra. \\
\hline Puente & Intestino, vísceras. \\
\hline Fijarse & Situación complicada, desorden. \\
\hline Atormentar & $\begin{array}{l}\text { Días de vacaciones que se crean cuando a los } \\
\text { festivos se suman otros días adicionales (por eje } \\
\text { porque están entre dos festivos muy próximos). }\end{array}$ \\
\hline Follón & \begin{tabular}{l} 
Causar dolor, molestia, enfado. \\
\hline
\end{tabular} \\
\hline
\end{tabular}

Tabla 3. Ejercicio de vocabulario

Una vez realizadas las actividades que tienen que ver más estrictamente con la respuesta al saludo y la comprensión de la situación descrita en el vídeo, se podrían comentar en clase otras cuestiones interesantes relacionadas con el español coloquial que se observan en fragmento de vídeo $^{6}$, por ejemplo:

- convenciones de la transcripción que tienen que ver con fenómenos típicos para el registro coloquial (mayúsculas para la pronunciación enfática; cursiva para el discurso directo)

- cuestiones de fonética y pronunciación: joer, mirao, pa'l, pa' casa

- marcadores conversacionales: ¿sabes?, ¿eh?, ¿vale?, ¿no?, etc.

- vocabulario coloquial, palabras malsonantes (mierda, culo)

Actividad 3. ¿Qué tal? en los foros. En la tercera actividad se propone matizar la exageración humorística «a nadie le importa cómo estás». Se pretende que los alumnos reflexionen acerca la aceptabilidad de las diferentes respuestas según el contexto y la relación entre los hablantes. En esta actividad se trabaja con una selección de fragmentos de respuestas publicadas en dos foros de opinión en español en los que hablantes nativos debaten entre ellos sobre cómo se usa o se debería usar la pregunta ¿qué tal? Conviene decírselo a los alumnos para que vean que no solo los extranjeros tienen dudas a la hora de saludar.

\begin{tabular}{|l|l|}
\hline & ¿Qué respondes cuando alguien te pregunta “¿Qué tal (tu día)?’? \\
\hline A & $\begin{array}{l}\text { Generalmente, la respuesta más políticamente correcta al responder esa pregunta es: «Bien, } \\
\text { normal» sin entrar en mayores detalles; y aun cuando se haya tenido un día infernal. } \\
\text { Ahora bien, dependiendo del grado de cercanía o familiaridad que se tenga con el interlocutor, } \\
\text { se podrá responder de manera más sincera o con mayores detalles. }\left(\mathrm{Q}^{7}\right)\end{array}$ \\
\hline
\end{tabular}

\footnotetext{
${ }^{6}$ Se pueden encontrar numerosas ideas de explotación de corpus conversacionales coloquiales en el trabajo de Albelda (2009). Muchas de ellas se pueden aplicar también a este fragmento de vídeo, aunque no se trate de una conversación coloquial prototípica.

7 Las respuestas marcadas como Q se han extraído y adaptado ligeramente del foro Quora (en la dirección: $\quad$ https://es.quora.com/Qu\%C3\%A9-respondes-cuando-alguien-te-pregunta-Que-tal-tud\%C3\%ADa, consultado el 15 de abril de 2020).
} 


\begin{tabular}{|c|c|}
\hline B & $\begin{array}{l}\text { Depende. Si me siento bien y no pasó nada fuera de lo normal, diría: bien, todo normal como } \\
\text { siempre. Lo mismo si no me siento tan bien y no quiero que lo sepan. Si no me siento muy bien } \\
\text { y tengo confianza con esa persona, diría el motivo y comenzaríamos a charlar sobre ello. Y si } \\
\text { me pasó algo bueno fuera de lo normal, se lo contaría contento. (Q) }\end{array}$ \\
\hline $\mathrm{C}$ & $\begin{array}{l}\text { A la pregunta ¿qué tal? hay que responder siempre en positivo para llegar lo antes posible al } \\
\text { segundo nivel de la conversación, dado que la introducción es solo por cortesía retórica. Si } \\
\text { realmente a la otra persona le importa cómo estás tú, ya surgirá más adelante de otra manera } \\
\text { con otras palabras. (Q) }\end{array}$ \\
\hline $\mathrm{D}$ & Excepto con personas muy cercanas a mí, me suelo limitar a responder «bien». (Q) \\
\hline $\mathrm{E}$ & $\begin{array}{l}\text { Yo si alguien me pregunta que tal estoy, yo suelo responder «bien»o «mal» dependiendo de la } \\
\text { situación, y de la confianza que tenga con quien este hablando. }\left(\mathrm{M}^{8}\right)\end{array}$ \\
\hline $\mathrm{F}$ & $\begin{array}{l}\text { Hombre, si te lo pregunta un dependiente de la tienda, no le vas a contar toda tu vida, digo yo. } \\
\text { (M) }\end{array}$ \\
\hline
\end{tabular}

Tabla 4. El texto para la actividad «¿Qué tal? en los foros»

A la lectura del texto le sigue un ejercicio en el que los alumnos deben completar la información para crear una especie de guía de actuación frente a la pregunta ¿qué tal?

\section{GUÍA BREVE PARA CONTESTAR A LA PREGUNTA ¿QUÉ TAL?}

1. La respuesta depende mucho del grado de entre las personas.

2. Una respuesta breve será la más adecuada si hablas con

3. Cuando no hay mucha confianza, lo normal es contestar incluso si has tenido un mal día.

4. Puedes contestar con más sinceridad y dar más detalles si estás hablando con .................

5. Si la otra persona realmente quiere saber cómo estás

6. Si quieres que la conversación avance más rápido

Después de completar la Guía, conviene volver sobre la Tabla 2 y las respuestas iniciales de los alumnos. Después de realizar todas las actividades propuestas hasta aquí, deberían ser capaces de formular la conclusión de que realmente la respuesta correcta es «depende», pero no tanto de la salud o el estado anímico real del hablante (lo que sugieren los emoticonos). Lo que hay que valorar sobre todo es la situación y la relación que existe entre los hablantes.

Actividad 4. ¿Te ha pasado esto? Como actividad final, los alumnos describen en forma de una anécdota breve y anónima algún malentendido cultural que han experimentado. La anécdota no necesariamente tiene que estar relacionada con los saludos; pueden escribir también sobre otras costumbres españolas que se alejan de la norma cultural polaca (por ejemplo, los dos besos, la cercanía física, las despedidas largas, etc.). A continuación, las redacciones se recogen y se mezclan. Después, uno o varios de los alumnos las leen en voz alta. El resto del grupo trata de adivinar a quién le ha ocurrido cada situación. Seguramente, varios alumnos han tenido experiencias similares, por lo que surgirán comentarios e intercambios espontáneos que pueden aprovecharse para

\footnotetext{
${ }^{8}$ Las respuestas marcadas como $\mathrm{M}$ se han extraído y adaptado ligeramente del foro de juegos en línea Mediavida (https://www.mediavida.com/foro/off-topic/contestais-pregunta-tal-545169/2, consultado el 15 de abril de 2020).
} 
continuar con la discusión. Los temas que surjan de esta actividad pueden retomarse en otra clase relacionada con la competencia sociocultural.

\section{Conclusiones}

Las fórmulas de saludo en español acompañadas de (o sustituidas por) las preguntas por la salud causan problemas a los aprendices polacos porque, por una transferencia sociocultural de su lengua, tienden a interpretar estas preguntas de manera literal. Las actividades que se han diseñado aportan ideas de cómo paliar este problema desde el aula y prevenir los choques culturales y los malentendidos entre los aprendices y los hablantes nativos del español. Se trata de una secuencia ordenada y gradual de actividades orientadas a la reflexión intercultural y al desarrollo de la competencia sociocultural.

\section{Bibliografía}

Albelda MARCO, M. (2009). «La enseñanza de E/LE a través de corpus de conversaciones coloquiales». Taller en el XVIII Encuentro práctico de profesores de ELE (Barcelona). Recuperado el 10 de abril de 2020, de: http://www.encuentropractico.com/recursos.html\#pragmatica.

García Wiedemann, E. J. y Moya Corral, J. (1994). «Las fórmulas de saludo en ELE». En J. Sánchez Lobato y I. Santos Gargallo (coords.), Problemas y métodos en la enseñanza del español como lengua extranjera: actas del IV Congreso Internacional de ASELE (Asociación para la Enseñanza del Español como Lengua Extranjera) (pp. 399-412). Madrid: SGEL.

Goffman, E. (1979). Relaciones en público. Microestudios del orden público. Madrid: Alianza Editorial.

JAWORSKI, A. (1994). "Pragmatic Failure in a Second Language. Greeting Responses in English by Polish Students», IRAL - International Review of Applied Linguistics in Language Teaching, 32 (1), pp. 41-56.

MARCJANIK, M. (2013 [2007]). Grzeczność w komunikacji językowej. Warszawa: Wydawnictwo Naukowe PWN SA.

MiQuel, L. (2004 [1997]). «Lengua y cultura desde una perspectiva pragmática: algunos ejemplos aplicados al español», redELE 2 (sin paginación).

MORENO FERNÁNDEZ, F. (1986). «Sociolingüística de los rituales de acceso en una comunidad rural», LEA: Lingüistica Española Actual, 8 (2), pp. 245-286.

PAlion-Musiol, A. y TAtoJ, C. (2016). «Propuestas didácticas para la enseñanza de la interculturalidad en la clase de ELE para los polacos». En J. Wilk-Racięska, A. Szyndler y C. Tatoj (eds.), Relecturas y nuevos horizontes en los estudios hispánicos. Vol. 4: Lingüística y didáctica de la lengua española (pp. 274-286). Katowice: Wydawnictwo Uniwersytetu Śląskiego.

ZIELIŃSKI, A. (2018). «Forms of greetings in Polish and Spanish», Romanica Cracoviensia, $18(2)$, pp. 105-114. 